\title{
An array of options for scaling up electrophysiology
}

\author{
New probes are letting neuroscientists record neuronal activity over larger areas and longer time periods
}

\author{
Michael Eisenstein
}

\section{A} single electrophysiology probe can intercept electrical signals reflecting the chatter between neurons at a discrete site within the brain. Although this is highly informative about local neuronal activity, many behaviors and neurological functions rely on extensive networks of cells that span multiple different brain structures. Trying to decipher these processes with individual probes is like trying to follow the action of a soccer match while only watching one corner of the field.

Loren Frank of the University of California at San Francisco cites his own struggles in investigating how memories are encoded and subsequently recalled to guide decision-making. "Memories are a prototypical example of something that isn't in one place in the brain-they involve neurons being activated all over the place," he says. "And so the question is: how can we understand that if we can't measure it?"

One could insert more probes, but this is a labor-intensive process that creates additional burdens for the animal for only modest levels of additional insight. "It can be extremely time-consuming, careful, and difficult work to get more than a few tens of neurons simultaneously," says Stanford University researcher Lisa Giocomo. Sophisticated imaging techniques offer another alternative, including fluorescent indicators that reveal activity in large numbers of individual neurons based on calcium flux or the release of neurotransmitters. But imaging is challenging for continuous, long-term studies in awake, behaving animals, and can only access the outermost layers of neural tissue.

Fortunately, a new generation of array-based probes is giving scientists a powerful new tool for addressing tough but essential neuroscience questions. These designs, which can interrogate hundreds or even thousands of neurons at once, make it possible to measure neuronal activity at the scale of circuits and networks spanning multiple brain structures in active animals. "It's possible to look much more in depth

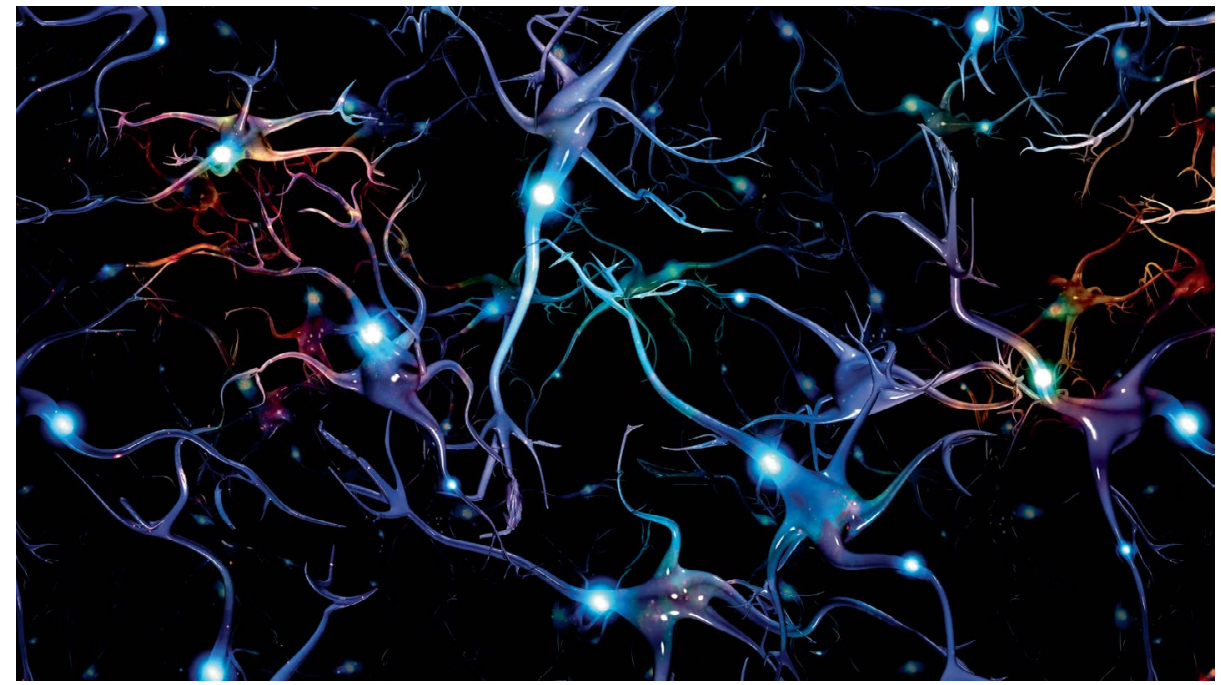

Researchers are improving probes that can pick up on the chatter between neurons across the brain. Credit: Bruce Rolff/Stocktrek Images/Getty

as well as in large areas," says György Buzsáki, of the New York University School of Medicine. "This is a real paradigm shift-I would say a new Renaissance-in electrophysiology"”

\section{Modernizing the 'Michigan probe'} Before joining the Howard Hughes Medical Institute's Janelia Research Campus in 2008, Timothy Harris worked at Bell Labs-an engineering mecca and birthplace of pivotal technologies such as the laser. Accordingly, when he began talking with Janelia's neuroscientists about their electrophysiology work, he was startled by the antiquated tools they were relying on. "It was literally 1960s technology," says Harris, referring to the 'Michigan probes' that were - and remain in widespread use.

These probes, developed by Kensall Wise at the University of Michigan, feature a needle-like silicon 'shank' lined with multiple, discrete recording sites. These probes can provide information about signaling events from multiple cells at different points along the probe, giving researchers insights into the organization of local neuronal networks. This is extremely useful, but Harris was intrigued by the idea of drawing on $21^{\text {st }}$ century micro-electronics manufacturing techniques to fabricate dense, miniaturized arrays that comprise many such probes in parallel, coupled to microscale electronics in a compact bundle that can be stably affixed to an animal's head.

In close collaboration with colleagues at University College London (UCL), the Allen Brain Institute, and IMEC-a state-of-the-art Belgian electronics fabrication facility - this concept was finally realized in 2017. The resulting 'NeuroPixels' device features probe shanks that each contain 384 sites that can independently detect nearby neuronal firing activity, coupled directly to signal-processing electronics at the base of the probe ${ }^{1}$. "You have the whole recording system right there," says University of Washington researcher Nicholas Steinmetz, who is part 


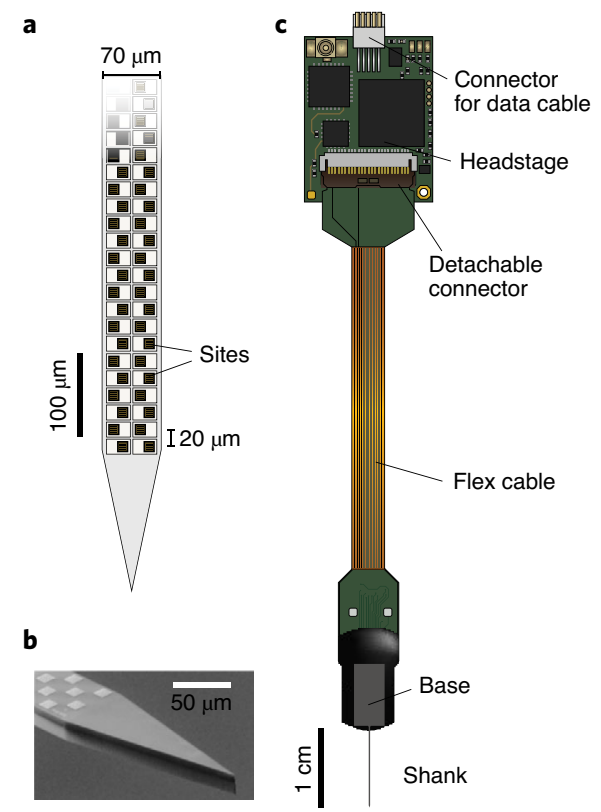

The first-generation Neuropixel probe. Reprinted with permission from Jun et al (2017)'. Springer Nature.

of the NeuroPixels consortium. "It really opened up this possibility of recording not just one area, but dozens of areas simultaneously, including both individual neurons and individual neuron populations in those areas."

They have proven particularly powerful for studying learning and memory, processes in which complex information may be relayed across far-flung sites in the brain. In a 2019 study $^{2}$, Steinmetz and his UCL colleagues studied the interplay between visual activity and decision-making in head-fixed mice that received a reward for successfully discriminating the level of contrast in two different image stimuli. By implanting multiple NeuroPixels shanks into ten mice, they were able to monitor the activity of tens of thousands of neurons in 42 different brain structures over the course of this task.

Giocomo's lab has also been making use of NeuroPixels in a series of studies that test how the mouse brain encodes information related to location and navigation into memory within a structure called the entorhinal cortex. These probes have allowed her to study the activity of several hundred neurons at a time as head-fixed mice 'travel' within a virtual-reality (VR) maze towards a destination where the animals can expect a water reward ${ }^{3}$. "In the dark, we found that there are these neurons that code for certain distances the animal travels," she says. "But when you turn the VR on, these neurons also get tethered to landmarks." These results gave new insights into how the entorhinal cortex integrates different kinds of information to generate internal 'maps'.

Today, NeuroPixels probes manufactured at IMEC are widely available to the research community, and Harris estimates that more than 5,500 probes have been shipped to over 400 labs worldwide. But the consortium has also recently announced the development of NeuroPixels version $2.0^{4}$-a smaller, more lightweight system that can record from up to 768 sites per shank, with as many as four shanks on a single probe. "We shrank the whole thing by a factor of three," says Harris, "[and] 1,500 channels on 16 shanks in a head-fixed mouse has now become a routine experiment." However, this upgrade version will not be generally available until mid-2022.

\section{A flexible solution}

Several groups have taken a different approach to array development, focusing on what they see as another critical limitation of the Michigan-style probes-their rigidity. Frank compares the resulting mismatch between the mechanical properties of the soft neural tissue and the stiff probe shank to sticking a knife into a wobbly gelatin dessert. This difference may not pose a problem in short-term studies or if the animal is secured through a head-fixation procedure, but such probes will inflict irreversible damage as time goes by. "Eventually, you will see scar tissue encapsulating the probe and the recording degrades," says Rice University neuroengineer Chong Xie.

One solution is to go fully non-invasive, as the Buzsáki group did with their NeuroGrid system - an ultra-thin polymer sheet containing hundreds of electrodes, which can be affixed onto the surface of the brain without actually penetrating the tissue. This array features hundreds of electrodes that can detect both individual neuron spikes as well as the local field potential signatures of groups of neurons at depths of up to 150-200 microns below the brain's surface. In 2017, Buzsáki's team used NeuroGrid to eavesdrop on communication between the hippocampus and the neocortex, which facilitates the long-term encoding of information from short-term memory ${ }^{5}$. They identified distinct 'ripple' patterns of brain activity that each of these structures use to encode data, and observed how these ripples become synchronized during learning. "The strength of the coupling was related to the strength of learning," says Buzsáki, adding that other groups have subsequently demonstrated the same synchronization in the human brain.
Other flexible arrays are more invasive, but enable access to the full volume of the rodent brain. As a postdoc in Charles Lieber's lab at Harvard University, Guosong Hong helped develop compressible mesh-like probes that can be introduced into the brain via injection through a needle $e^{6}$. Once unfurled in the tissue, these polymer-based meshes provide numerous windows that can be penetrated by neurons and other brain cells, anchoring the probes firmly in place and allowing the implanted site to regenerate. Each probe contains 64 electrodes, which can collectively detect activity from up to 200 neurons at a time, and the mechanical properties of the mesh closely match those of the brain, such that minimal scarring occurs over time. "We were actually able to measure single-neuron activity from the probe over the course of almost a year without seeing decay of the signal," says Hong, now at Stanford University. Hong's team is using these for lifelong studies of learning and memory that would otherwise be impossible with rigid electrodes. "We can track how neural circuits involved with this behavior change as a function of age in both wild-type and Alzheimer's model animals," he says.

For the Frank laboratory, the priority was to develop a system that enables the monitoring of large numbers of neurons in multiple, widely distributed regions of the brain. Drawing on foundational polymer-based electrode research from the Lawrence Livermore National Laboratoryinitially developed for an artificial retina project-Frank's team devised soft, biocompatible shanks that each contain up to 128 recording channels. Multiple of these can be implanted at different sites, with all the electrophysiological recordings collected and processed at a single,

lightweight device mounted to the animal's head. "We've been looking at what happens when an animal makes a memory-guided decision," says Frank. "So how is activity in the hippocampus related to activity in the prefrontal cortex, and we are now able to do that at a level where we're getting hundreds of cells from both regions at the same time." In some recent experiments, his group has been able to analyze up to four different brain structures in a single rat.

\section{Picking a probe}

The choice of which design is the best fit comes down to a number of factors, including the area of the brain being investigated. For the NeuroPixels probes, for example, the electrode channels are arranged in a line along the length of the shank, which makes them best suited for sampling structures that lie along a single axis. 
This is ideal for some tissues, as Giocomo notes from her work with the entorhinal cortex. "It's a long thin structure, and the NeuroPixels probes are long and thin, so that's been fine," she says. But some cortical regions and other areas of the brain, such as the hippocampus, have a more complicated structure that is more challenging to study with a linear probe.

More generally, the hippocampus has posed a daunting challenge for both rigid and flexible probe arrays. For example, the Frank lab's multi-site electrophysiology studies still rely on traditional 'tetrode' wire electrodes to monitor this particular structure. "The hippocampus turns out to be a very fragile flower," he says, noting that its densely-packed neurons are easily damaged or killed during shank implantation. To address this, he is now collaborating with the Xie lab, which has devised micron-thick, ultra-flexible polymer electrodes that generate minimal perturbation after being implanted in the brain 7 . "The surrounding cells don't feel the forces from the deformation of these electrodes," says Xie. "And we can go pretty much anywhere in the rodent brain."

Experiments with freely-moving animals could also pose a challenge for rigid probe arrays, especially if those experiments run for extended periods of time. Several groups have demonstrated the successful use of NeuroPixels in freely-moving mice for up to weeks at a time, but most users are currently opting to apply the system in the context of head-fixed experiments, like Giocomo's VR-based studies. "It's a bit clunky for a mouse," says Harris. "People do it, but it's a stretch." This is another problem NeuroPixels 2.0 aims to address, with a smaller form factor and software that can effectively compensate for the movement of the probe in the brain of an active animal. But for the time being, flexible probes may offer a more lightweight and stable alternative for some studies. Hong and Frank have each found that their probe systems offer minimal interference even for studies that offer considerable physical activity. "We had a task where the animal ran on a two-meter track, 750 meters to a kilometer a day," says Frank. "We were still able to follow individual neurons for more than a week."

But on the other hand, NeuroPixels is much more accessible to new users, with considerable documentation, training and support available. Furthermore, the surgical implantation process should not pose a serious challenge to users familiar with electrophysiology experiments using standard Michigan probes. In contrast, flexible electrode implantation requires time, skill, and patience. Hong notes that his mesh electrodes can crumple into a useless mess if injected too fast, and his group makes use of a computer-controlled injection system that slowly withdraws the needle as the meshwork is released into the tissue.

Xie and Frank are both making use of rigid 'shuttle' devices to deliver their flexible electrodes into the brain, which are then gradually withdrawn to leave just the probe behind, but this becomes labor-intensive as one begins to introduce large arrays of such probes. "Each surgery takes up to 10 hours to do," says Xie. "It's not something that's going to happen every day in every lab." Frank notes that his group recently received a National Institutes of Health grant to refine their probe technology, and he is hoping to begin moving towards commercial development of a more robust and user-friendly version of their flexible arrays.

\section{A broadening array}

Although the recording of brain activity in mice and rats has been the natural first step for testing out these various array probes, the range of applications has grown rapidly. The NeuroPixels team has developed probes that are particularly well-suited for the large brains of nonhuman primates, and several groups have already performed large-scale neural recording experiments in these species-as well as more exotic models. "There was a paper I saw where they were putting NeuroPixels in a species called the brown ghost knifefish, and getting these beautiful recordings from the midbrain of this fish," says Steinmetz. The NeuroGrid system has also been applied to a variety of large animal species, but Buzsáki is particularly interested in translating the device to clinical applications, and has already shown that this system can effectively home in on seizure-related patterns of neuronal activity in epileptic patients ${ }^{8}$.

There is also considerable excitement around the idea of being able to record the network-level effects of targeted perturbations in neural circuits with methods like optogenetics. In such experiments, certain neurons are genetically modified to express channels that either activate or inhibit firing in response to specific wavelengths of light, and several groups are devising strategies to combine

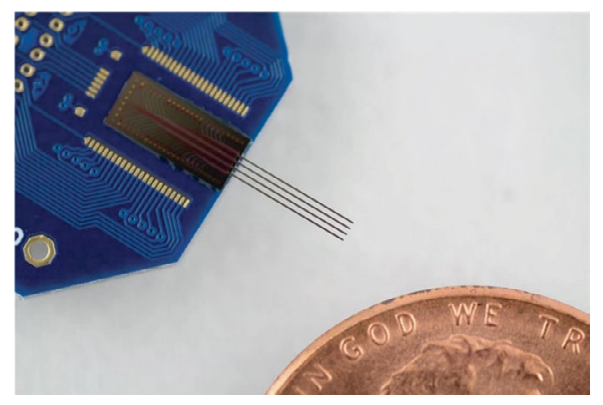

The MiniSTAR optoelectrode, capable of recording and manipulating neuronal activity. Reprinted with permission from Kim et al. (2020) ${ }^{9}$. Springer Nature.

their probes with such targeted illumination. For example, Buzsáki and his collaborator Eusik Yoon at the University of Michigan have developed neuron-sized microscale LEDs that can both manipulate and record the activity of individual cells". "The next generation will have 64 or even 128 sites where you can write in and read out from the brain," says Buzsáki.

But perhaps the most intriguing question is just how much high-resolution neuronal activity can realistically be captured in a single experiment-and relatedly, how much information neuroscientists need to capture to get an accurate view of brain function. And even as NeuroPixels 2.0 moves towards formal release, Harris is already thinking about the next big leap forward. "I'm simply focused on the art of the possible," he says. "I think we can get to 25,000 neurons in the mouse-so that's what we'll do, and then we'll ask to what degree we understand the total activity."

Michael Eisenstein ${ }^{凶}$

Freelance science writer, Philadelphia, PA, USA.

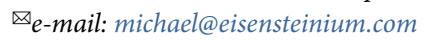

Published online: 15 April 2021

https://doi.org/10.1038/s41684-021-00758-3

References

1. Jun, J. J. et al. Nature 551, 232-236 (2017).

2. Steinmetz, N. A., Zatka-Haas, P., Carandini, M. \& Harris, K. D. Nature 576, 266-273 (2019).

3. Campbell, M. G., Attinger, A., Ocko, S. A., Ganguli, S. \& Giacomo, L. M. bioRxiv https://doi.org/10.1101/2020.10.05.327106 (2021).

4. Steimetz, N. A. et al. bioRxiv https://doi. org/10.1101/2020.10.27.358291 (2021).

5. Khodagholy, D., Gelinas, J. N. \& Buzsáki, G. Science 358, 369-372 (2017).

6. Fu, T. et al. Nat Method 13, 875-882 (2016).

7. Luan, L. et al. Sci. Adv. 3, el601966 (2017).

8. Khodagholy, D. et al. Sci. Adv. 2, el60127 (2016).

9. Kim, K. et al. Nat Commun 11, 2063 (2020). 